\title{
AVALIAÇÃO DA DOR COMO QUINTO SINAL VITAL: UMA ESCOLHA PROFISSIONAL DE INTERVENÇÃO FISIOTERAPÊUTICA
}

\section{PAIN ASSESSMENT AS A FIFTH VITAL SIGN IN PHYSICAL THERAPY}

\author{
Ana Cláudia Heinen*, Cássia da Luz Goulart**, Ana Cristina Sudbrack***, Tânia Cristina \\ Malezan Fleig***, Andréa Lúcia Gonçalves da Silva***
}

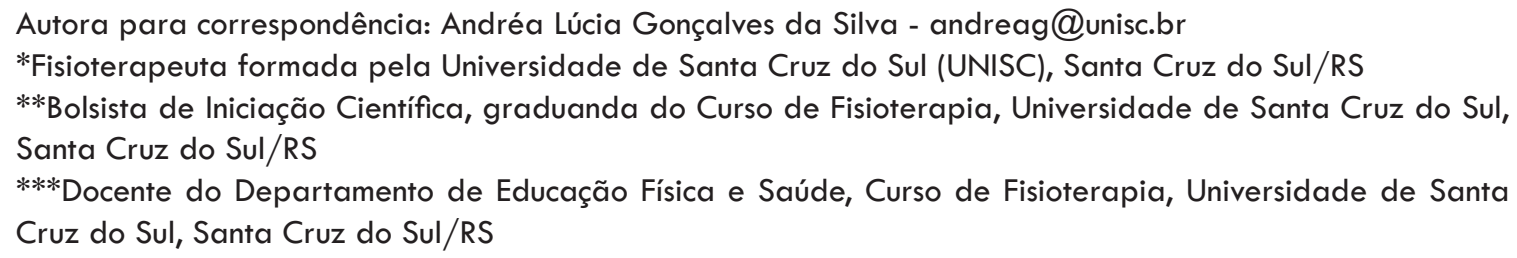

\section{R E S U M O}

Introdução: A dor é tão universal que seu reconhecimento deve ser uma habilidade essencial dos profissionais da saúde. Por ser considerada subjetiva e expressa de diferentes formas, foram criadas várias escalas para mensurar a dor do indivíduo dentro do processo de avaliação. Objetivo: reconhecer a escala de dor que melhor se aplica na avaliação deste sintoma junto aos fisioterapeutas e pacientes para consequente implementação no serviço de fisioterapia hospitalar. Métodos: estudo transversal, tipo quali-quantitativo, com amostra de conveniência composta por fisioterapeutas e pacientes adultos hospitalizados no Hospital Santa Cruz, de Santa Cruz do Sul-RS. Constituído de quatro etapas: aplicação de questionário junto aos fisioterapeutas; aplicação de questionário junto aos pacientes; utilização da escala de dor pelos fisioterapeutas; nova aplicação de questionário junto aos fisioterapeutas. Resultados: No questionário aplicado junto aos fisioterapeutas ( $\mathrm{n}=15)$, obteve-se a escala numérica como a preferida por ser de fácil entendimento $(53,33 \%)$ e com melhor relação custo-benefício $(53,33 \%)$. Já aplicação com os pacientes $(n=23)$, houve predomínio da escala visual analógica (EVA) (teste $=39,13 \%$; re-teste $=43,48 \%$ ). A EVA foi implementada junto ao serviço de fisioterapia hospitalar por 08 fisioterapeutas nas suas avaliações e atendimentos. No acompanhamento da utilização desta, em registro nos prontuários, verificou-se o uso mais efetivo nas avaliações e por fisioterapeutas. Conclusão: Foi possível verificar o reconhecimento da escala de dor que melhor se aplica na avaliação deste sintoma como quinto sinal vital, bem como reconhecer junto a fisioterapeutas e pacientes a mais indicada quanto à compreensão e fácil entendimento, ou seja, a Escala EVA.

Palavras-chave: Avaliação; Dor; Fisioterapia. 
Introduction: The pain is so universal that recognition should be an essential ability of health professionals. For being considered subjective and expressed in different ways, several scales were designed to measure the pain of the individual within the evaluation process. Aim: To recognize which pain scale best applies in the pain assessment by physiotherapists for its implementation in a hospital physiotherapy service. Methods: It is a qualitative and quantitative transversal study, with a convenience sample of physiotherapists and adult patients hospitalized in the Hospital Santa Cruz, Santa Cruz do Sul-RS. It consisted of four steps: applying a questionnaire to the physiotherapists; applying a questionnaire to the patients; using the pain by physiotherapists scale; new application questionnaire with the physiotherapists. Results: the questionnaire addressed to the physiotherapists $(n=15)$ obtained the numerical scale as the favorite because it was easier to understand (53.33\%) and more cost-effective $(53.33 \%)$. In applying the questionnaire to the patients $(n=23)$ they preferred the visual analogue scale (VAS) because it was considered to be easier to understand (pratical test $=39.13 \%$, test $=43.48 \%$ ). The VAS was implemented in the hospital physiotherapy service by 08 physiotherapists in their evaluation and treatments. In monitoring the use of VAS, in the physiotherapist records, it was their most effective use of evaluation and by physiotherapists. Conclusions: It was possible to verify the recognition of the pain scale that best applies in the assessment of this symptom as the fifth vital sign, as well as recognition from the physiotherapists and patients the most appropriate as to understand, that is, the VAS.

Keywords: Evaluation; Pain; Physical Therapy 


\section{INTRODUÇÃO}

O profissional fisioterapeuta se insere nas mais diferentes áreas de atuação utilizando-se de procedimentos, técnicas, metodologias e abordagens específicas $^{1,2}$, baseadas prioritariamente nas queixas que $\circ$ paciente apresenta, muitas delas relacionadas à sua patologia, sendo a principal e mais difícil de ser conduzida, a dor. Sua presença ou ausência são determinantes no atendimento fisioterapêutico, podendo ser este um limitante, pois muitas vezes, impossibilita a realização da terapêutica adequada ao caso clínico do paciente.

A dor é tão universal que seu reconhecimento deve ser uma habilidade essencial de todos àqueles que trabalham na área da saúde com sujeitos saudáveis ou não. Sua presença é uma sobrecarga na vida cotidiana, limitando a capacidade funcional e prejudicando a qualidade de vida. A International association for the study of pain (IASP) define a dor como experiência sensorial e emocional associada ao sofrimento psicológico e dano de tecido ${ }^{3-5}$. A dor pode ser considerada subjetiva e expressa de forma diferente sendo que o primeiro desafio é sua mensuração ${ }^{3}$. Nesse intuito foram criadas escalas para mensurar a dor do indivíduo e assim realizar uma intervenção. Entretanto, é necessário escolher a escala que melhor se enquadra ao paciente ${ }^{3}$.

O processo de avaliação da dor é amplo e envolve a obtenção de informações relacionadas à data de início, localização, intensidade e duração dos episódios dolorosos, às qualidades sensoriais e efetivas do paciente, aos fatores que iniciam, aumentam ou diminuem sua intensidade ${ }^{6-8}$. O indivíduo hospitalizado possui fatores que influenciam diretamente o estado psicológico, podendo alterar ou intensificar a representação de dor. No entanto, quando o paciente consegue se comunicar fica fácil para o profissional identificar o que está afetando?. Desta forma, as manifestações de dor devem ser reconhecidas e interpretadas, para que o sofrimento causado nas pessoas em caráter de vulnerabilidade também possa ser atenuado ${ }^{3}$.

Sendo assim, o alívio da dor é um requisito para que $\circ$ paciente obtenha uma ótima recuperação e qualidade de vida ${ }^{5}$. Existem vários tipos de escalas que podem ser fotocopiadas e utilizadas para auxiliar às avaliações, para monitorar o progresso e avaliar o efeito do tratamento. $O$ uso em hospitais, na comunidade e nos lares de assistência propõese a detectar a dor, aumentando a probabilidade de atendimento de alta qualidade para idosos e reduzindo a morbidade associada à dor ${ }^{4}$.

Este estudo foi conduzido para responder ao reconhecimento, dentre as escalas de Dor, daquela que melhor representa $\circ$ quinto sinal vital na avaliação e tratamento fisioterapêutico do paciente hospitalizado, pontuando a efetividade do plano terapêutico. Sendo que sua implementação junto a um serviço de fisioterapia tem grande importância sobre o plano de tratamento, visto que perante limiares muito altos de dor $\circ$ profissional pode deixar de intervir, ou modificar sua terapêutica de acordo com as necessidades reais e momentâneas do paciente.

Para isto, estabeleceram-se objetivos no intuito de reconhecer a escala de dor preferenciados pelos fisioterapeutas, reconhecendo a mais indicada e de fácil aplicação, bem como junto aos pacientes a escala de dor que corresponde ao seu fácil entendimento.

\section{MÉTODOS}

Estudo transversal, quali-quantitativo. A amostra deste estudo é aleatória de conveniência, composta por fisioterapeutas atuantes no ambiente hospitalar e pacientes adultos hospitalizados no Hospital Santa Cruz, de Santa Cruz do Sul-RS. Foram incluídos no estudo todos os fisioterapeutas do hospital Santa Cruz , de ambos o sexo, faixa etária, tempo e área de atuação profissional, bem como independente do conhecimento e utilização prévio de escalas de dor e assistência de pacientes em quadros álgicos. Foram excluídos os fisioterapeutas que se recusaram a participar da pesquisa e/ou a assinar o termo de consentimento livre esclarecido. A inclusão dos pacientes obedeceu alguns critérios: maiores de 18 anos, nível de consciência lúcido e comunicativo, 
independente de uso prévio de escala de dor, que concordaram em participar do estudo através da assinatura do Termo de Consentimento Livre e Esclarecido e que apresentavam quadro álgico agudo ou crônico independente da intensidade. Foram excluídos os pacientes fazendo uso de opióides e sedativos. O projeto de pesquisa foi apreciado e aprovado pelo Comitê de Ética em
Pesquisa (CEP-UNISC) sob protocolo de n ${ }^{\circ} 2447 / 09$. Após a busca das escalas de aferição de dor na literatura, foram selecionadas sete escalas: escala de cores, escala linear analógica visual, escala visual analógica - EVA, escala verbal, escala de faces, escala analógica visual de faces, escala linear analógica não visual - numérica (FIGURA 1).

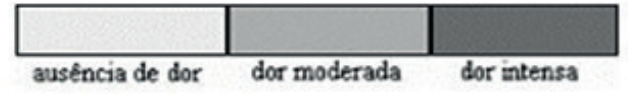

A: Escala de Cores

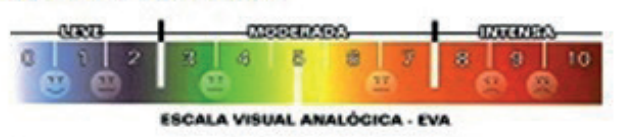

C: Escala Visual Analógica - EVA

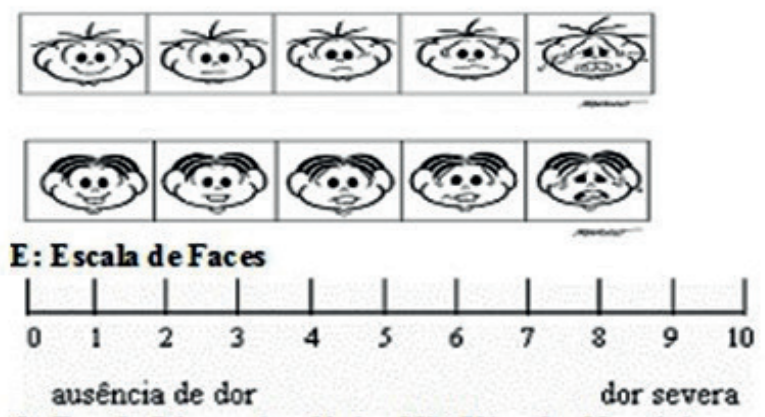

G: Escala Linear Analógica Não Visual - Numérica

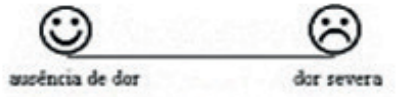

\section{B: Escala Linear Analógica Visual}

0-Sem Dor

1-Dor Ligeira

2-Dor Moderada

3- Dor Intensa

4-Dor Máxima

D: E scala Verbal

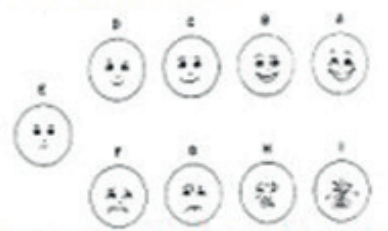

F: Escala Analógica Visual de Faces

H: Não se Aplica

Figura 1. Escalas para aferição de dor. A: escala de cores, B: escala linear analógica visual, C: EVA, D: escala verbal, E: escala de faces, F: escala analógica visual de faces, G: escala linear analógica não visual - numérica. H: Não se aplica.

Através da escala de cores é possível que os pacientes possam identificar a intensidade da dor, utilizando mais a sua intuição, podendo assim localizar a dor em um esquema corporal e posteriormente, colorir - local com a cor que mais expresse sua dor?. A escala linear analógica visual é indicada por uma linha reta, como ausência de dor e do lado oposto, a maior intensidade de dor ${ }^{8}$. Pela EVA, é preciso que haja o contato visual do paciente com a escala e ele deve ser capaz de apontar ou sinalizar ao examinador em que grau sua dor está ${ }^{9}$, já a escala verbal objetiva a mensuração da intensidade da dor, em contextos clínicos com valores numéricos?. A escala de faces e a escala analógica visual de faces são mais utilizadas em crianças, compostas por expressões, as quais variam da expressão sem dor até a dor insuportável ${ }^{9}$. Por fim, a escala linear analógica não visual-numérica, é quantificada pela intensidade dolorosa através de escores que variam de zero até dez, sendo essa caracterizada por dor leve, intensa, aguda ou muito intensa ${ }^{9}$. Após definir as escalas, o estudo constou de 04 etapas de investigação (Figura 2).

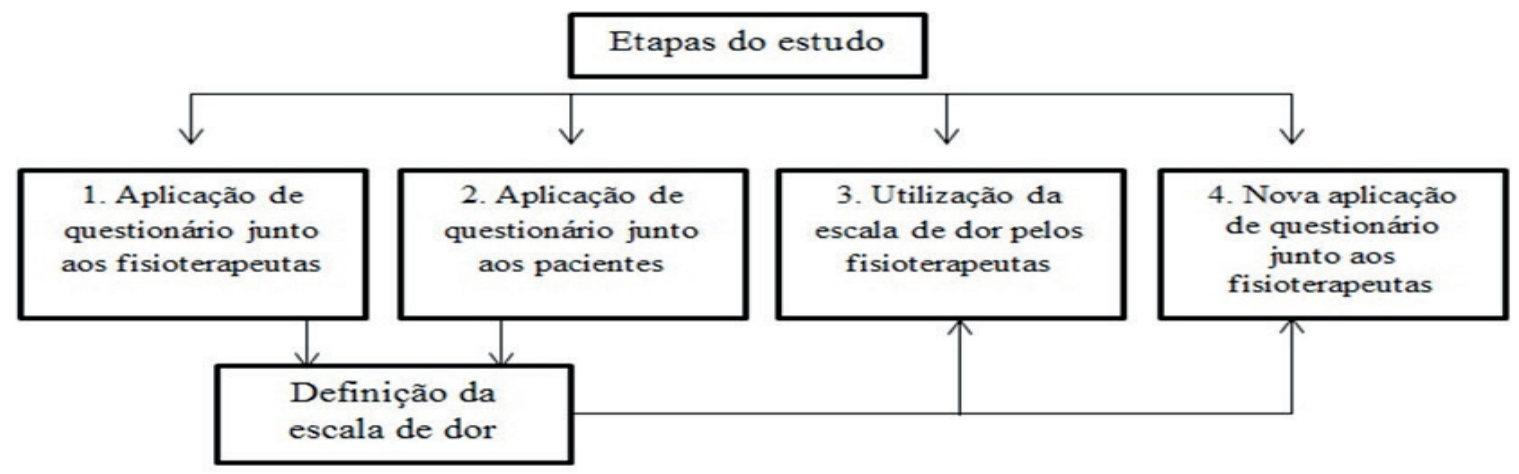

Figura 2. Etapas de investigação do estudo. 
Na primeira etapa foi realizada a pesquisa com fisioterapeutas atuantes na área hospitalar, no intuito de calibração do roteiro de investigação e reconhecimento da indicação da escala de dor que mais se aplica para a atuação profissional neste ambiente. Após o conhecimento das escalas os fisioterapeutas responderam as questões visualizadas no Quadro 1.

Quadro 1. Questões aplicadas junto aos fisioterapeutas para estudo piloto e determinação da melhor escala de avaliação da dor.

\begin{tabular}{|l|l|}
\hline 1. & $\begin{array}{l}\text { Em sua opinião qual escala é mais adequada para o paciente adulto } \\
\text { hospitalizado? }\end{array}$ \\
\hline 2. & $\begin{array}{l}\text { Em sua prática profissional, qual seria a escala mais fácil para os pacientes } \\
\text { hospitalizados responderem? }\end{array}$ \\
\hline 3. & $\begin{array}{l}\text { Com sua experiência profissional, qual seria a escala mais rápida de ser } \\
\text { aplicada? }\end{array}$ \\
\hline 4. & $\begin{array}{l}\text { Dentre as escalas escolhidas acima, eleja apenas uma na qual teremos melhor } \\
\text { relação custo-benefício? }\end{array}$ \\
\hline 5. & Você utiliza algum outro instrumento de avaliação da dor não esteja indicada \\
na Figura 1? Quale
\end{tabular}

As escalas de dor contidas no roteiro de investigação dos fisioterapeutas foram também aplicadas junto aos pacientes hospitalizados, compondo a segunda etapa de investigação, com um único objetivo: os pacientes deveriam indicar qual a sua escala de preferência para identificar a sua dor, no que tange a facilidade de entendimento da mesma. Junto a estes indivíduos foi realizado teste e re-teste, em dias subsequentes, para assegurar os resultados e minimizar o viés de memória.

Posteriormente, de posse dos resultados, fez-se a análise das escolhas de ambos os grupos de sujeitos, identificou-se a escala de maior frequência de resposta e instituiu-se uma única escala para utilização dos fisioterapeutas atuantes no ambiente hospitalizado, compondo a terceira etapa do estudo. Para que isto fosse possível, foi estabelecido um encontro com a profissional responsável pelo serviço, onde foram explicados os objetivos do trabalho e acordado que os fisioterapeutas iriam programar a utilização da referida escala em todos os seus atendimentos, inclusive na avaliação, mediante termo de consentimento. Após este momento foi realizado acompanhamento dos prontuários dos pacientes, três vezes por semana, para verificar a aplicabilidade da escala de dor e coletar de todas as informações relevantes ao estudo. $O$ acompanhamento foi realizado durante o período de um mês, sendo que após este foi realizada a quarta etapa da pesquisa com a reaplicação do questionário junto aos fisioterapeutas, agora com adaptações referentes à escala eleita e implementada no serviço de fisioterapia, conforme podemos ver no Quadro 2.

Quadro 2. Adaptações realizadas no questionário inicial (Quadro 1) para avaliação da escala de dor EVA pelos fisioterapeutas.

\begin{tabular}{|l|l|}
\hline 1. & $\begin{array}{l}\text { Em sua opinião esta escala (EVA) é adequada para o paciente adulto } \\
\text { hospitalizado? Justifique. }\end{array}$ \\
\hline 2. & $\begin{array}{l}\text { Em sua prática profissional, escala visual analógica (EVA) é fácil para os } \\
\text { pacientes hospitalizados responderem? }\end{array}$ \\
\hline 3. & $\begin{array}{l}\text { Com sua experiência profissional, a escala visual analógica é rápida de ser } \\
\text { aplicada? }\end{array}$ \\
\hline 4. & $\begin{array}{l}\text { A escala visual analógica tem uma boa relação custo-benefício na avaliação da } \\
\text { dor? }\end{array}$ \\
\hline 5. & Você utiliza outra escala que não seja a escala visual analógica (EVA)? Qual? \\
\hline 6. & O uso da escala visual analógica (EVA) interferiu nos objetivos e condutas? \\
\hline
\end{tabular}




\section{RESULTADOS}

\section{Escolha do Instrumento de Avaliação da Dor} Esta etapa da pesquisa foi composta por 01 grupo de fisioterapeutas ( $n=15)$, e os resultados do questionário aplicado junto aos fisioterapeutas estão descritos na Figura 3.

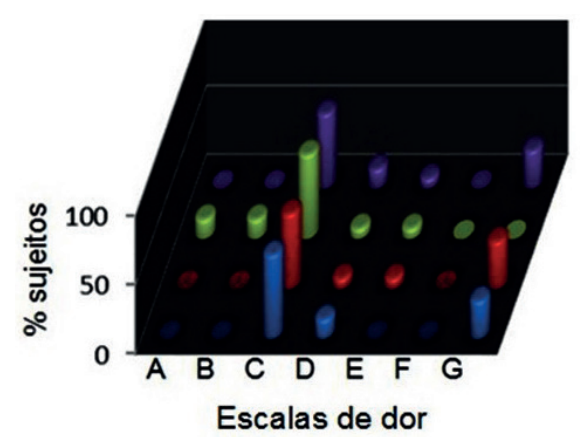

घQuestão 1

घQuestão 2

घQuestão 3

घQuestão 4

Figura 3. Instrumento de avaliação da dor eleito pelos fisioterapeutas do serviço hospitalar. \%: frequência de sujeitos; A: escala de cores, B: escala linear analógica visual, C: EVA, D: escala verbal, E: escala de faces, F: escala analógica visual de faces, G: escala linear analógica não visual - numérica. Na questão 5 do questionário nenhum

fisioterapeuta aplicava outro instrumento para avaliar dor.

Tanto na questão que aborda a opinião do profissional a respeito da escala mais adequada para $\circ$ paciente hospitalizado quanto na que diz respeito à facilidade de entendimento por parte dos pacientes, pode-se verificar que 60 e 53,33\% dos profissionais respectivamente, acreditam que a escala mais adequada seja a escala EVA.

Nesta fase, também se avaliou um grupo de pacientes $(n=23)$, e como resultado do teste e reteste, aplicando-se as mesmas escalas de dor, pode-se verificar, que no primeiro teste $39,13 \%$ dos pacientes apresentaram preferência na escala EVA, sendo esta confirmada no re-teste com $43,48 \%$ (FIGURA 4).

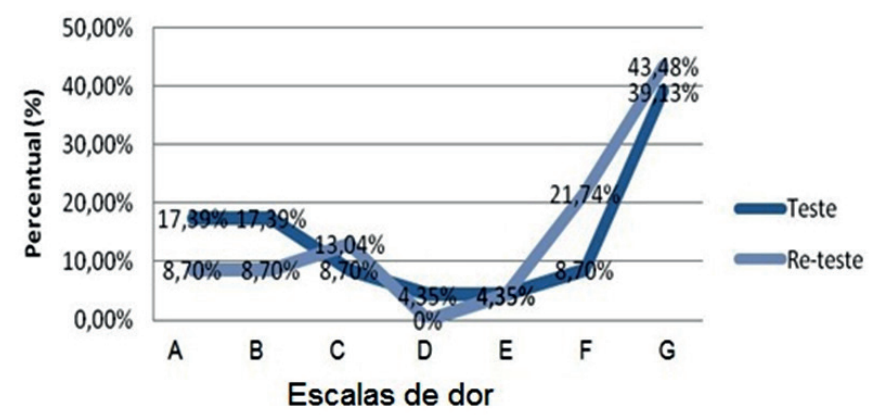

Figura 4. Instrumento de avaliação da dor eleito pelos pacientes do estudo no teste e re-teste. \%: frequência de sujeitos; $A$ : escala de cores, B: escala linear analógica visual, C: escala linear analógica não visual - numérica, D: escala verbal, E: escala de faces, F: escala analógica visual de faces, G: EVA.
Implementação do Instrumento de Avaliação da

\section{Dor- Escala EVA}

As características clínicas dos pacientes estão descritas na Tabela 01, com maior frequência do sexo masculino, idade adulta $60,7 \pm 10,5$ anos e intervenção fisioterapêutica ortopédica.

Tabela 01. Caracterização clínica dos pacientes hospitalizados que participaram do estudo.

\begin{tabular}{lc}
\hline \multicolumn{1}{c}{ Variáveis } & $\mathrm{N}=63$ \\
\hline Sexo, n(\%) & $36(57,1)$ \\
Masculino & $27(42,9)$ \\
Feminino & \\
Intervenção Fisioterapêutica, (\%) & 38,1 \\
Ortopédicas & 23,8 \\
Neurológicas & 22,2 \\
Cardiorrespiratórias & 2,7 \\
Digestórias & 3,1 \\
Outras & \\
\hline Dados expressos em frequência (\%), média e desvio padrão.
\end{tabular}

Pertinente à utilização da escala de dor, em um total de 29 avaliações e 234 atendimentos fisioterapêuticos, em apenas $4,27 \%$ e $6,41 \%$ foi utilizada a escala EVA respectivamente (FIGURA 5).

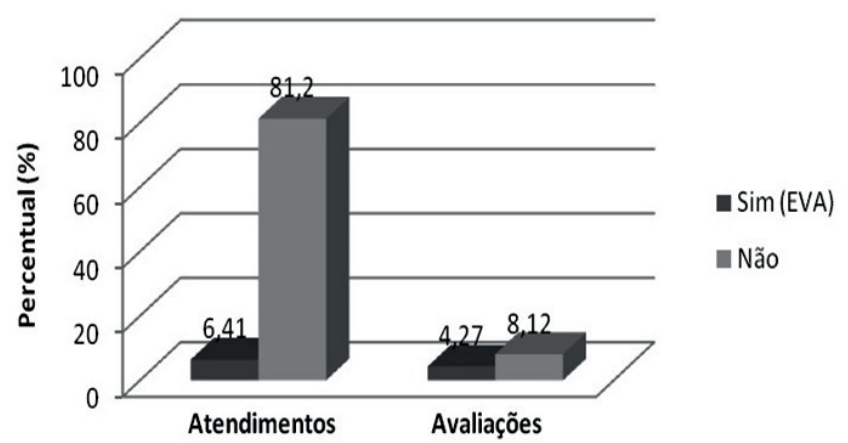

Figura 5. Demonstração das situações (Atendimento ou Avaliação) onde a Escala EVA foi utilizada pelos fisioterapeutas participantes do estudo, junto aos pacientes hospitalizados, em comparação com a não utilização da mesma.

Visto que os fisioterapeutas que atendem durante a semana fazem um acompanhamento sistemático e diário da evolução dos pacientes sob sua supervisão, pode-se remeter a menor assiduidade quanto ao uso da escala, à autoconfiança, que faz com que não necessariamente seja feita uma avaliação diária de todas as variáveis.

\section{DISCUSSÃO}

Foi possível verificar o reconhecimento da escala de dor que melhor se aplica na avaliação deste sintoma como quinto sinal vital, bem como reconhecer junto a 
fisioterapeutas e pacientes a mais indicada quanto à compreensão e fácil entendimento, ou seja, a Escala EVA. A escolha e implementação se deu pelo fato de possuir múltiplas possibilidades de identificação do sintoma, confirmando-se com os relatos de pacientes e fisioterapeutas. A escala visual analógica é de fácil compreensão ao paciente, é provida de instruções claras e conceitos simples, permitindo aplicação rápida com intervenção mínima. Afirmação esta que vai ao encontro das falas dos pacientes durante a testagem das escalas, onde eles relataram que o fato da escala EVA apresentar múltiplas possibilidades de identificar a dor (faces, números, cores, descrição em leve/moderada/intensa) facilita a comunicação entre terapeuta e paciente ${ }^{10}$.

Acredita-se que a escala EVA foi à escolhida pelos pacientes e fisioterapeutas, em virtude de sua aplicabilidade possibilitar a tomada de decisões importantes no que se refere à dor e ainda proporcionar, com maior exatidão, a quantificação da dor que está sentindo e se a terapêutica que está sendo utilizada realmente é eficaz no tratamento ${ }^{8}$.

A intensidade da dor, as diferentes escalas apresentam alto grau de validade e confiabilidade e devem ser utilizadas, pois a avaliação baseada exclusivamente na observação dos cuidadores frequentemente subestima a real intensidade da dor $^{10}$. Conforme Freitas et al. (2009) ${ }^{11}$, as escalas unidimensionais de dor, nas quais o paciente é questionado para descrever um aspecto de sua dor ou do seu tratamento, são instrumentos utilizados para quantificá-la e sua estrutura pode possuir várias formas ${ }^{11}$. Diante da escolha do paciente, optou-se por implementação da escala EVA na segunda etapa da pesquisa.

A avaliação da dor e o registro sistemático e periódico de sua intensidade são fundamentais para que se acompanhe a evolução dos pacientes e se realize 0 ajuste necessário ao tratamento ${ }^{12}$. Pedroso e Celich $(2006)^{13}$ relatam que a dor é considerada um sinal vital tão importante quanto os outros e deve sempre ser avaliada em ambiente clínico, para se compreender um tratamento ou conduta terapêutica, sendo que sua eficácia com o seguimento depende de uma avaliação e mensuração da dor confiável e válida. Sendo assim, o controle da dor é uma atribuição de todo profissional de saúde ${ }^{14}$. Conceitos fundamentais a respeito dos mecanismos de dor e de suas repercussões nas áreas física, emocional e social dos indivíduos, das possibilidades diagnósticas e sobre princípios da terapêutica devem ser conhecidas por todos aqueles que atuam em qualquer campo da área das ciências da saúde ${ }^{14}$. É provável que tenhamos que quebrar muitos paradigmas de nossa prática, principalmente com procedimentos como a inserção nas equipes, a flexibilidade, a subjetivação da dor, o acolhimento à família e a comunicação terapêutica ${ }^{15}$.

Algumas limitações podem ser observadas em nosso estudo, tais como o número amostral de fisioterapeutas e a diversidade de pacientes que não nos permite muitas inferências e como perspectivas futuras, sugere-se avaliar a eficácia do tratamento fisioterapêutico sobre melhora da dor através da EVA.

\section{CONCLUSÃO}

Foi possível verificar o reconhecimento da escala de dor que melhor se aplica na avaliação deste sintoma como quinto sinal vital, bem como reconhecer junto aos fisioterapeutas e pacientes a mais indicada quanto à compreensão e fácil entendimento, ou seja, a Escala EVA. A inclusão da avaliação da dor como quinto sinal vital poderá assegurar que os pacientes tenham acesso à intervenção para o seu controle, garantindo, desta forma, o seu bem-estar.

\section{REFERÊNCIAS}

1. COFFITO. Conselho Federal de Fisioterapia e Terapia Ocupacional, 2014. Disponível em: http:// www.coffito.org.br

2. Sahrmann SA. Diagnosis by the Physical Therapist: a prerequisite for treatment. Phys. Ther. 1988;68(11):1703-06.

3. Ciena AP, Gatto R, Pacini VC, Picanço VV, Magno IMN, Loth EA. Influência da intensidade da dor sobre as respostas nas escalas 
unidimensionais de mensuração da dor em uma população de idosos e de adultos jovens. Ciências Biológicas e da Saúde. 2008;29(2):201 - 12. doi: $10.5433 / 1679-0367.2008 v 29 n 2$ p201

4. Vitor AO, Ponte EL, Soares PM, Carvalho KM, Rodrigues MES, Patrocínio MCA et al. Psicofisiologia da dor: uma revisão bibliográfica. R. Eletr. de Com. Inf. Inov. Saúde. 2008;2(1):87-96. doi: 10.3395/ reciis.v2i1.133pt

5. Calil AM, Pimenta CAM. Intensidade da dor e adequação de analgesia. Rev. Latino-Am. Enfermagem. 2005;13(5):692-699. doi: 10.1590/ S0104-11692005000500013

6. Barbosa TP, Beccaria LM, Pereira RAM. Avaliação da experiência de dor pós-operatória em pacientes de unidade de terapia intensiva. Rev Bras Ter Intensiva. 2011 ; 23(4):470-7. Doi: $10.1590 /$ S0103-507X2011000400012

7. Saça CS, Carmo FA, Arbuleia JPS, Souza RCX, Alves SA, Rosa BA. A dor como 50 sinal vital: atuação da equipe de enfermagem no hospital privado com gestão do Sistema Único de Saúde. J Health Sci Inst. 2010; 28(1):35-41.

8. Bottega FH, Fontana RT. A dor como quinto sinal vital: utilização da escala de avaliação por enfermeiros de um hospital geral. Texto \& contexto enfermagem. 2010;19(2):283-90. doi: 10.1590/ s0104-07072010000200009

9. Fortunato JGS, Furtado MS, Hirabae LFA, Oliveira JS. Escalas de dor no paciente crítico: Uma revisão integrativa. Revista HUPE. 2013;12(3): 110 117. doi: $10.12957 /$ rhupe.2013.7538

10. Augusto ACC, Soares CPSS, Resende MA, Pereira LSM. Avaliação da dor em idosos com doença de Alzheimer: uma revisão bibliográfica. Textos Envelhecimento. 2004;7(1):21-5.

11. Freitas CC, Vieira PR, Torres, GVB; Pereira, CRA. Avaliação da dor com o uso de escalas unidimensionais. Rev. Dor. 2009;10(1):56-62.

12. Almeida NAM; Souza JT; Bachion MM, Silveira, NA. Utilização de técnicas de respiração e relaxamento para alívio de dor e ansiedade no processo de parturição. Rev. Latino-Am. Enfermagem. 2005; 13(1):52-58. doi: 10.1590/ S0104-11692005000100009

13. Fernandes CR, Gomes JMA, Moraes RP, Marinho DS, Holanda MA, Oliveira FRA. Avaliação sistemática do delirium e da dor em pacientes criticamente enfermos. Rev. Dor. 2009;10(2): 158168.

14. Pedroso RA, Celich, KLS. Dor: quinto sinal vital, um desafio para o cuidar em enfermagem. Texto contex enferm. 2006; 15(2):270-276. doi: $10.1590 /$ S0104-07072006000200011

15. Rodrigues I, Zago M. Cuidados paliativos: realidade ou utopia? Ciênc. Cuid. Saúde. 2009;8(supl):1 36-141. doi: 10.4025/ cienccuidsaude.v8i0.9740 